\title{
HIROSHIMA UNIVERSITY RADIOCARBON DATES I WEST AND SOUTH COASTS OF SRI LANKA*
}

\author{
JINADASA KATUPOTHA** \\ Department of Geography, Hiroshima University, 1-1-89, Higashi \\ Senda Machi, Naka-ku, Hiroshima 730, Japan
}

The following list reports radiocarbon dates of geologic samples collected from the west, southwest and south coasts of Sri Lanka during August 1985. The tidal range on Sri Lanka coasts is very small, the lowest neap at $-0.48 \mathrm{~m}$ and highest spring at $+0.52 \mathrm{~m}$ from msl (data based on the standard point of Colombo, datum level $0.38 \mathrm{~m}$ below msl).

Radiocarbon age measurements were carried out at the Department of Geography, Hiroshima University Radiocarbon Dating Laboratory. The dates were obtained by liquid scintillation counting of methanol using a laboratory procedure (Fujiwara \& Nakata, 1984). All coral, shell, and calcareous algae samples were treated in $10 \% \mathrm{HCl}$, and pure $\mathrm{CaCO}_{3}$ samples were converted into methanol. Dates are correlated with the chronology of the Holocene epoch (Fairbridge, 1968).

The results are expressed in radiocarbon years relative to $\mathrm{AD} 1950$ based on the Libby half-life of $5568 \pm 30$ years, using new NBS oxalic acid standard (SRM 4900C) as "modern" (Stuiver, 1983).

\section{ACKNOWLEDGMENTS}

This research involves a preliminary data collection and pertains to the author's doctorate program at the Department of Geography, University of Hiroshima, sponsored by the Ministry of Education, Japan. I am indebted to my supervisor, Kenzo Fujiwara, for his direct guidance in sample collection techniques. Grateful thanks are due Takashi Nakata who helped me with the laboratory procedures. I also wish to thank Marcus Karunanayake, Department of Geography, University of Sri Jayewardenepura, Sri Lanka, for his great assistance in my field work, and P A Pirazzoli, CNRS-Intergeo, Paris, France, for reviewing the manuscript and making valuable comments.

\section{GEOLOGIC SAMPLES}

\section{West Coast series}

Two samples from exposed beach rock, W of Negombo $\left(7^{\circ} 12^{\prime} 30^{\prime \prime} \mathrm{N}\right.$, $79^{\circ} 49^{\prime} 00^{\prime \prime} \mathrm{E}$ ), Negombo dist. Samples dated as Late Holocene (Late Subboreal).

\footnotetext{
* A contribution to the project IGCP-200 "Sea-Level Correlations and Applications" ** Address from March 1988: Department of Geography, University of Sri Jayewardenepura, Gangodawila, Nugegoda, Sri Lanka
} 
HR-125. Negombo

Calcareous algae from surface of beach rock, elev $0.25 \mathrm{~m}$, approx above high tide level. Comment: date indicates that one stage of coastal progradation had begun after Late Holocene (Late Subboreal).

HR-126. Negombo

$3460 \pm 160$

Marine shell from same loc as HR-125. Comment: same as HR-125.

\section{Southwest Coast series}

Eleven samples from well-developed buried coral beds, raised coral reef patches and a dug well, Galle dist. Dates correlated with Middle Holocene (Main Atlantic) and Late Holocene (Early Subboreal, Late Subboreal, and Early Subatlantic). Stratigraphic sequences and deposition pattern of branching (Acropora) and massive (Porites) corals (stood upright) and shells of buried beds (coral quarry nos. 1 and 2) mostly appear in growth position. Relatively good condition of materials reveals that accumulation was not caused by catastrophic event such as storm wave action.

HR-111. Dimbulduwa-Akurala

$5830 \pm 90$

Coral (Acropora) from coral quarry no. $1\left(6^{\circ} 12^{\prime} 10^{\prime \prime} \mathrm{N}, 80^{\circ} 03^{\prime} 57^{\prime \prime} \mathrm{E}\right)$, depth $1.40 \mathrm{~m}$ below msl.

HR-112. Dimbulduwa-Akurala

$5820 \pm 90$

Coral (Echinopora) from coral quarry no. 1, at same loc as HR-111, depth $2 \mathrm{~m}$ below msl.

HR-113. Dimbulduwa-Akurala

$5910 \pm 80$

Coral (Porites) from coral quarry no. 1, at same loc as HR-111, depth $2.90 \mathrm{~m}$ below msl.

HR-114. Dimbulduwa-Akurala

$6000 \pm 90$

Coral (Acropora) from coral quarry no. 1, at same loc as HR-111, depth $3 \mathrm{~m}$ below msl.

HR-115a. Wellemeda-Akurala

Super modern

Shell from coral quarry no. $2\left(6^{\circ} 11^{\prime} 50^{\prime \prime} \mathrm{N}, 80^{\circ} 03^{\prime} 57^{\prime \prime} \mathrm{E}\right)$, depth $0.25 \mathrm{~m}$ below msl.

HR-115b. Wellemeda-Akurala

$\mathbf{5 8 0 0} \pm \mathbf{8 0}$

Coral (Acropora) from quarry no. 2, at same loc as HR-115a, depth $2.10 \mathrm{~m}$ below msl.

HR-109. Wellemeda-Akurala

$6110 \pm 80$

Coral (Acropora) from coral quarry no. 2, at same loc as HR-115a, depth $3.90 \mathrm{~m}$ below msl.

General Comment: dated sequences of coral quarry nos. 1 and 2 reveal that branching corals and massive corals developed inland as isolated patches following marine transgression during Holocene. The author agrees with 
suggestions made by Deraniyagala (1958, p 15), and Neef and Veeh (1981).

HR-110. Habaraduwa

$5560 \pm 70$

Coral (Acropora) from coral quarry no. $3\left(5^{\circ} 59^{\prime} 50^{\prime \prime} \mathrm{N}, 80^{\circ} 17^{\prime} 00^{\prime \prime} \mathrm{E}\right)$, depth $0.90 \mathrm{~m}$ to $1.10 \mathrm{~m}$ below msl. Comment: date correlates with coral quarry nos. 1 and 2.

HR-121. Galle-Unawatuna

$1960 \pm 60$

Coral (Acropora) from dug well $\left(6^{\circ} 00^{\prime} 30^{\prime \prime} \mathrm{N}, 80^{\circ} 14^{\prime} 50^{\prime \prime} \mathrm{E}\right)$, depth $0.96 \mathrm{~m}$, approx below msl. Comment: partly rounded coral may be displaced by wave action.

HR-117. Akurala-Telwatte

$4070 \pm 80$

Coral (Goniastrea) from emerged reef patch, in growth position $\left(6^{\circ} 10^{\prime}\right.$ $10^{\prime \prime} \mathrm{N}, 80^{\circ} 03^{\prime} 50^{\prime \prime} \mathrm{E}$ ), elev $0.40 \mathrm{~m}$ to $0.60 \mathrm{~m}$ above msl. Comment: date confirms that previous sea level was higher than at present between Middle Holocene (Main Atlantic) and Late Holocene (Early Subboreal) in Sri Lanka. The author agrees with suggestions of Shepard (1961; see Hubbs, Bean \& Suess, 1962, p 212).

HR-116. Hikkaduwa

$5100 \pm 70$

Coral (Goniastrea) from emerged reef patch, in growth position cemented with sandstone $\left(6^{\circ} 07^{\prime} 45^{\prime \prime} \mathrm{N}, 80^{\circ} 05^{\prime} 45^{\prime \prime} \mathrm{E}\right)$, elev $0.51 \mathrm{~m}$ above msl. Comment: same as HR-117.

\section{South Coast series}

Three samples from two shell middens and one sample from emerged shell bed of lake margins, Hambantota dist. Emerged shell bed dated as Middle Holocene (Main Atlantic).

\section{HR-124. Hungama}

$5780 \pm 110$

Marine shells (Veneridae) from dry lagoon bed at Kalametiya Lake $\left(6^{\circ}\right.$ $05^{\prime} 30^{\prime \prime} \mathrm{N}, 80^{\circ} 55^{\prime} 30^{\prime \prime} \mathrm{E}$ ), elev $1.31 \mathrm{~m}$ above msl. Comment: the author infers that varieties of shell belonging to marine form living in inter-tidal zone were deposited preceding coastal emergence after Middle Holocene (Early Subboreal).

HR-123. Karagan Lewaya

$3050 \pm 100$

Shells from midden in coastal sand dune close to Karagan Lake $\left(6^{\circ} 07^{\prime}\right.$ $20^{\prime \prime} \mathrm{N}, 80^{\circ} 05^{\prime} 10^{\prime \prime} \mathrm{E}$ ), elev $2.28 \mathrm{~m}$ above msl. Comment: shell midden consists of uniform species of marine shells (Veneridae) forming layer several $\mathrm{cm}$ thick. Date suggests inhabitants dwelled along coastal low hilly areas adjoining lagoons prior to Late Holocene (Early Subboreal).

HR-122. Udamalala

$4050 \pm 50$

Shells (Veneridae) from midden of lake margin $\left(6^{\circ} 10^{\prime} 15^{\prime \prime} \mathrm{N}, 80^{\circ} 10^{\prime} 00^{\prime \prime}\right.$ E), elev 5.25m approx msl. Comment: same as HR-123. 


\section{REFERENCES}

Deraniyagala, P E P, 1958, The Pleistocene of Ceylon: Colombo, Ceylon Natl Mus Pub, p 10 15.

Fairbridge, R W, 1968, Holocene, Postglacial or Recent epoch: The encyclopedia of geomorphology: New York, Reinhold Book Corp, $533 \mathrm{p}$.

Fujiwara, K and Nakata, T, 1984, Methanol liquid scintillation radiocarbon dating (1): Bull Faculty Lit, Univ Hiroshima, v 44, p 120-134 (in Japanese with English abs).

Neef, G, and Veeh, H H, 1981, A Holocene coral reef in southwestern Sri Lanka: Ceylon Jour Sci (Bio Sci), v 14, no. 1\&2, p 116-119.

Hubbs, C L, Bean, G S and Suess, H E, 1962, La Jolla natural radiocarbon measurements II: Radiocarbon, v 4, p 204-238.

Shepard, P F, 1961, Sea level rise during the past 20,000 years: Zeitschr Geomorphol NF supp 3 , p 30-35.

Stuiver, M, 1983, International agreements and the use of the new oxalic acid standard, in Stuiver, $\mathrm{M}$ and Kra, R S, eds, Internatl ${ }^{14} \mathrm{C}$ conf, 11 th, Proc: Radiocarbon, v 25, no. 2, $\mathrm{P}$ 793-795. 